\title{
A rare case of Candida prosthetic joint infection: Diagnostic and therapeutic challenges in a resource poor country.
}

\author{
WAMP Samaranayake ${ }^{1}$ \\ Sri Lankan Journal of Infectious Diseases 2018 Vol.8 (1):51-54 \\ DOI: http://dx.doi.org/10.4038/sljid.v8i1.8156
}

\begin{abstract}
Candida infection of knee prostheses is rare but increasingly reported. This report describes a candida prosthetic joint infection in a healthy woman treated with antifungal therapy following removal of knee prosthesis in a Teaching Hospital in Sri Lanka.
\end{abstract}

Keywords: Candida spp., Prosthetic joint infection, Antifungal therapy.

\section{Introduction}

Prosthetic joint infections due to Candida species are extremely uncommon and most are caused by Candida albicans followed by Candida parapsilosis. ${ }^{1}$ It is commonly caused via direct inoculation of the organism or due to transient candidaemia, while underlying host immune suppression acts as the main predisposing factor. ${ }^{2}$ Clinical recognition is often difficult due to its indolent onset and lack of other symptoms of infection during the initial stage. Definitive diagnosis is made by isolation of the causative agent from multiple samples taken intra-operatively. Inadequacy of clinical evidence of improvement during management poses a therapeutic challenge and published case reports vary widely in outcome and therapeutic approach. ${ }^{3}$

This report describes one patient seen at the orthopaedic unit of a Teaching Hospital in Sri Lanka with prosthetic joint infection due to Candida spp. treated with antifungal drugs following removal of prosthesis. Diagnostic and therapeutic challenges during her management which contributed towards the poor outcome are discussed.

\section{Case Report}

A 63-year-old healthy female underwent total left knee replacement in January 2013 followed by right knee replacement in August 2014 for severe osteoarthritis. Two weeks after the latter, she developed pain and effusion in her right knee joint for which ten days of intravenous meropenem was given, to which she responded. She had no history of diabetes mellitus or prolonged antibiotic therapy and was not immunosuppressed.

${ }^{1}$ Teaching Hospital, Batticaloa, Eastern Province, Sri Lanka.

Address for correspondence: Dr WAMP Samaranayake, No 57/17, Railway Station Road, Veyangoda, Sri

Lanka. Tel: +94772955484 Email: manorisamaranayake1981@gmail.com

(i) http://orcid.org/0000-0001-5279-9166.

Received 9 October 2017 and revised version accepted 26 March 2018

(c) (7)

This an open-access article distributed under the terms of the Creative Commons Attribution License, which permits unrestricted use, distribution, and reproduction in any medium, provided the original author and source are credited. 
One year after surgery, she presented to the hospital with fever, pain, moderate swelling and decreased range of motion in her right limb. She had an elevated erythrocyte sedimentation rate (ESR) of $60 \mathrm{~mm} /$ one hour and C reactive protein (CRP) of $24 \mathrm{mg} / \mathrm{L}$ levels with a normal white blood count. X-ray of the knee showed bone destruction around the prosthesis suggestive of septic loosening. Bone scan showed evidence of an active inflammatory process in the right lower femur and right upper tibia. Joint fluid and blood culture were negative. Joint fluid aspirate was turbid and full report showed raised protein of $5.5 \mathrm{~g} / \mathrm{dl}$ (normal level 2.5-3 g/dl) with neutrophils $400 / \mathrm{mm}^{3}$, lymphocytes $110 / \mathrm{mm}^{3}$ and RBC $2440 / \mathrm{mm}^{3}$. She was treated with intravenous meropenem and vancomycin for 7 days and discharged.

Pain over her right knee joint was continuous for which she undertook native treatment intermittently. She was readmitted with unbearable pain in 2017. On admission, she was afebrile with an ESR of $92 \mathrm{~mm} /$ one hour and CRP of $265 \mathrm{mg} / \mathrm{L}$ with normal white blood count (total count 8800 and differential count: 58\% neutrophils, 37\% lymphocytes). Blood culture was negative. Complete removal of the right knee joint prosthesis was carried out and direct examinations showed yeast cells. Culture of debrided bone, joint fluid and peri-prosthetic tissue grew Candida spp. (germ tube negative) in pure growth. Histology of bone particles showed chronic granulomatous inflammation, but no yeast cells were seen. Disc diffusion method of antifungal susceptibility testing showed that the isolate was susceptible to amphotericin with no zone of inhibition to fluconazole and voriconazole. No minimum inhibitory concentrations of antifungals (MIC) were performed.

The patient was started on intravenous amphotericin B deoxycholate $1 \mathrm{mg} / \mathrm{kg}$ once daily. A good therapeutic response with dramatic reduction of ESR and CRP were observed after a week of amphotericin B deoxycholate. However, she developed severe infusion related side effects (fever and chills). It was decided to switch to anidulafungin (100mg twice a day for one day followed by $100 \mathrm{mg}$ daily) from intravenous amphotericin B deoxycholate for a minimum period of 6 weeks. There was an undue delay in initiation of anidulafungin for three weeks. Meanwhile, she succumbed to the infection.

The timeline of the patient's clinical course is given below.

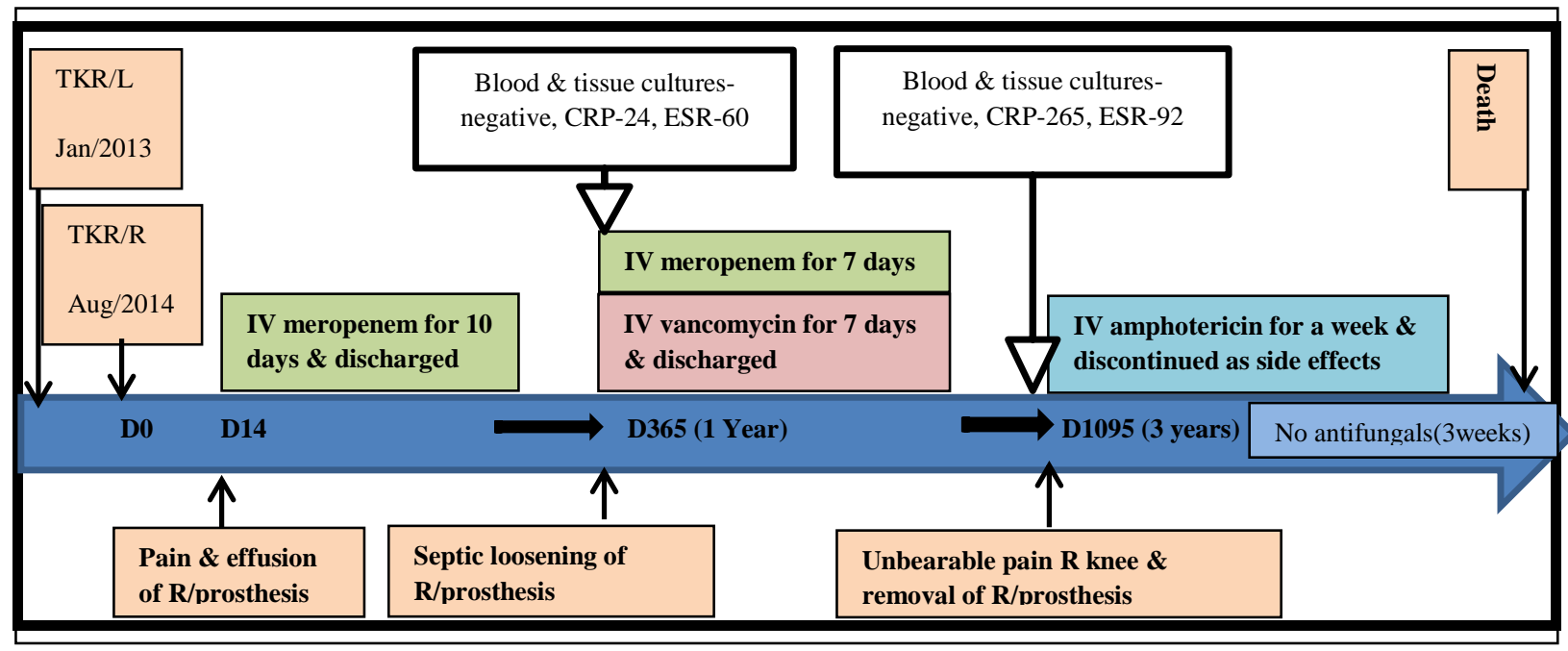

\section{Discussion}


Prosthetic joint infection (PJI) due to Candida species is still a rare phenomenon. However, cases are reported increasingly due to rising number of joint arthroplasties worldwide. ${ }^{1}$ Underlying immunocompromised status, systemic illness (diabetes mellitus), the use of antineoplastic agents, prolonged use of antibiotics and indwelling catheters are some of the risk factors for fungal PJI. ${ }^{2}$ Despite this, nearly half the cases occur in patients without any identifiable risk factor ${ }^{3}$ as in this patient.

Pain and swelling are the main symptoms of PJI due to Candida species, although the onset of symptoms can be insidious, and development of the disease can be slow ${ }^{2}$ as seen in this patient. Most of the reported cases do not have systemic fungal disease. Most authors recommend obtaining multiple samples, prolonged incubation of cultures, special staining and histological examination for laboratory diagnosis. The diagnosis was made through clinical and radiological findings along with isolation of Candida spp from multiple intraoperative bone and tissue specimens in this patient.

The cornerstone of diagnosis remains isolation and speciation of fungus along with in vitro susceptibility testing. ${ }^{3,4}$ The susceptibility of Candida spp. to available antifungal agents is generally predictable if the species of the infecting isolate is known. ${ }^{5,6}$ Clinical and Laboratory Standard Institute (CLSI) and European Committee on Antimicrobial Susceptibility Testing (EUCAST) have developed susceptibility testing of yeasts against most, but not all antifungal drugs which is an essential tool to guide treatment.

C. albicans is the most common cause for fungal PJI reported in the literature followed by $C$. parapsilosis and other non-albicans spp. ${ }^{2}$ The majority of $C$. albicans infections are associated with biofilm formation on the host or on the surfaces of medical devices or prostheses leading to resistance to fluconazole. ${ }^{2}$ Candida spp. isolated from our patient was found to be a germ tube negative but speciation was not carried out. Antifungal sensitivity testing showed sensitivity to amphotericin B with resistance to fluconazole and voriconazole. Choice of antifungal treatment was made based on results obtained and availability of antifungal agents.

The long-term antifungal use with two-stage exchange arthroplasty is currently regarded as the gold standard to eradicate the infection which showed the highest success rate $(85 \%){ }^{4}$ However, the ideal interval between implant removal and re-implantation, the usefulness of antifungal-loaded cement spacers and the type, duration of systemic antifungal treatment are still controversial. ${ }^{1}$ Amphotericin B is the gold standard, but is highly nephrotoxic and may not be useful for long term administration. ${ }^{3}$ Lipid formulations of amphotericin B or fluconazole would be better options. A high bio-availability, extended half-life, absence of serious side effect and high concentration in joint fluid make fluconazole a better choice though some nonalbicans candida species are resistant to fluconazole. ${ }^{4,7}$ Echinocandins may be a good alternative due to its low toxicity, broad spectrum of activity or if the patient is intolerant to amphotericin B deoxycholate. The Infectious Diseases Society of America recommends treatment with antifungal for at least 6 weeks after removal of the arthroplasty while chronic suppression is recommended if removal of prosthesis is not an option. ${ }^{6,8,9,10}$ Our patient was treated with amphotericin B deoxycholate following removal of the infected prosthesis to which she responded clinically. However, she did not tolerate conventional amphotericin B deoxycholate for more than a week and it was decided to switch to anidulafungin based on the available literature. ${ }^{1,4,5,7,8}$ As it was an expensive and restricted drug, there was a considerable delay in obtaining the drug during which the patient was not on any anti-fungal treatment. She succumbed to the infection three weeks after amphotericin was stopped. 
Delays in diagnosis, lack of species identification with no MIC values and non-availability of newer antifungals compounded the difficulties experienced in the management of a rare cause of prosthetic joint infection in this patient. Collaboration between orthopaedic surgeons, radiologists, histopathologists and microbiologists is needed to improve outcomes in Sri Lanka. A multidisciplinary approach to determine risk and design strategies to maximize the use of available facilities would be helpful for earlier diagnosis and starting appropriate treatment.

\section{Informed consent}

The patient gave her informed verbal consent for the inclusion in this publication.

\section{Acknowledgement}

I would like to acknowledge the Orthopaedic team for their contribution in management of this patient.

\section{Conflict of Interest}

The author has no conflict of interest.

\section{References}

1. Jakobs O, Schoof B, Klatte TO, et al. Fungal periprosthetic joint infection in total knee arthroplasty: a systematic review Orthopedic Reviews 2014; 6:5623-28.

doi: https://doi.org/10.4081/or.2015.5623

2. Kojic EM, Darouiche RO. Candida infections of medical devices. Clin Microbiol Rev 2004; 17(2):255-267. doi: https://doi.org/10.1128/CMR.17.2.255-267.2004

3. Reddy KJ, Shah JD, Kale RV, et al .Fungal prosthetic joint infection after total knee arthroplasty. Indian J Orthop 2013; 47(5):526-529. doi: https://doi.org/10.4103/00195413.118213

4. Cobo F, Granger JR, Sampedro A, et al. Candida prosthetic joint infection. A review of treatment methods. J. Bone Joint Infect 2017; 2(2):114-121. doi: https://doi.org/10.7150/jbji.17699

5. Clinical laboratory Standard Institute. Zone Diameter Interpretive Standards, Corresponding Minimal Inhibitory Concentration (MIC) Interpretive Breakpoints, and Quality Control Limits for Antifungal Disk Diffusion Susceptibility Testing of Yeasts; Third Informational Supplement. CLSI document M44-S3. Wayne: Clinical and Laboratory Standards Institute, 2009

6. Osmon DR, Berbari EF, Anthony R, et al. Diagnosis and management of prosthetic joint infection: clinical practice guidelines by the Infectious Diseases Society of America. Clinical Infectious Diseases 2013; 56:1-25 doi: https://doi.org/10.1093/cid/cis803

7. Daniel RP. Candida hip prosthesis infection: A case report. Biomed J Sci \& Tech Res 2017; 1(2). doi: https://doi.org/10.26717/BJSTR.2017.01.000223

8. Paul J, White SH, Nicholls KM, et al. Prosthetic joint infection due to Candida parapsilosis in the UK: Case report and literature review. European J Clin Microbiol 1992; 11(9):847-849. doi: https://doi.org/10. 1007/BF01960889

9. Phelan DM, Osmon DR, Keating MR, et al. Reimplantation arthroplasty for candida prosthetic joint infection: A report of 4 cases and review of the literature. Clinical Infectious Diseases 2002; 34(7):930-8. doi: https:// doi.org/10.1086/339212

10. Pitchai S, Kanniyan K, Kumar P A, et al. Fungal periprosthetic joint infection by Candida glabrata - Two stage revision is a good option. IOSR Journal of Dental and Medical Sciences 2016; 15(12):68-70. doi: https://doi.org/10.9790/0853-1512046870 